\title{
Transport of paclitaxel (Taxol) across the blood-brain barrier in vitro and in vivo
}

\author{
Stephan Fellner, ${ }^{1}$ Björn Bauer, ${ }^{2}$ David S. Miller, ${ }^{3}$ Martina Schaffrik, ${ }^{1}$ Martina Fankhänel, ${ }^{1}$ \\ Thilo Spruß, ${ }^{1}$ Günther Bernhardt, ${ }^{1}$ Claudia Graeff, ${ }^{2}$ Lothar Färber, ${ }^{4}$ \\ Harald Gschaidmeier, ${ }^{4}$ Armin Buschauer, ${ }^{1}$ and Gert Fricker ${ }^{2}$
}

${ }^{1}$ Institute of Pharmacy, University of Regensburg, Regensburg, Germany

${ }^{2}$ Institute of Pharmaceutics and Biopharmacy, University of Heidelberg, Heidelberg, Germany

${ }^{3}$ Laboratory of Pharmacology and Chemistry, National Institute of Environmental Health Sciences, NIH,

Research Triangle Park, North Carolina, USA

${ }^{4}$ Novartis Pharma GmbH, Nürnberg, Germany

\begin{abstract}
Paclitaxel concentrations in the brain are very low after intravenous injection. Since paclitaxel is excluded from some tumors by p-glycoprotein (p-gp), the same mechanism may prevent entry into the brain. In vitro, paclitaxel transport was examined in capillaries from rat brains by confocal microscopy using BODIPY Fl-paclitaxel. Western blots and immunostaining demonstrated apical expression of p-gp in isolated endothelial cells, vessels, and tissue. Secretion of BODIPY Fl-paclitaxel into capillary lumens was specific and energy-dependent. Steady state luminal fluorescence significantly exceeded cellular fluorescence and was reduced by $\mathrm{NaCN}$, paclitaxel, and SDZ PSC-833 (valspodar), a p-gp blocker. Leukotriene $\mathrm{C}_{4}\left(\mathrm{LTC}_{4}\right)$, an Mrp2-substrate, had no effect. Luminal accumulation of NBDL-cyclosporin, a p-gp substrate, was inhibited by paclitaxel. In vivo, paclitaxel levels in the brain, liver, kidney, and plasma of nude mice were determined after intravenous injection. Co-administration of valspodar led to increased paclitaxel levels in brains compared to monotherapy. Therapeutic relevance was proven for nude mice with implanted intracerebral human U-118 MG glioblastoma. Whereas paclitaxel did not affect tumor volume, co-administration of paclitaxel (intravenous) and PSC833 (peroral) reduced tumor volume by $90 \%$. Thus, $\mathrm{p}$-gp is an important obstacle preventing paclitaxel entry into the brain, and inhibition of this transporter allows the drug to reach sensitive tumors within the CNS.
\end{abstract}

J. Clin. Invest. 110:1309-1318 (2002). doi:10.1172/JCI200215451.

\section{Introduction}

Paclitaxel (Taxol) and derivatives are active against various tumors (1-6) and have also been used to treat malignant glioma and brain metastases (7-9). However, brain tumors constitute a difficult problem and the therapeutic benefit of paclitaxel has been variable and low. This could be attributed to its limited entry into the CNS. Although paclitaxel is very lipophilic, concentrations in the CNS are very low after intravenous administration $(10,11)$.

Paclitaxel appears to be a substrate of the multidrug resistance protein $\mathrm{p}-\mathrm{gp}(12-14)$, and it is like-

Received for publication March 12, 2002, and accepted in revised form August 8, 2002.

Address correspondence to: Gert Fricker, Institut für Pharmazeutische Technologie und Biopharmazie, Im Neuenheimer Feld 366, 69120 Heidelberg, Germany. Phone: 49-6221-548336; Fax: 49-6221-545971;

E-mail: jw3@ix.urz.uni-heidelberg.de.

Portions of this work were presented at the Annual Meeting of the German Society of Pharmacology and Toxicology, Mainz, Germany, March 15-17, 2000, and the Annual Meeting of the German Pharmaceutical Society, Münster, Germany,

October 4-7, 2000.

Conflict of interest: No conflict of interest has been declared. Nonstandard abbreviations used: $p$-glycoprotein (p-gp); KrebsHenseleit buffer (KHB); enhanced chemiluminescence (ECL); PBS plus 0.04\% Triton X-100 (PBST); diaminobenzidine (DAB); room temperature (RT); solid-phase extraction (SPE); leukotriene $\mathrm{C}_{4}\left(\mathrm{LTC}_{4}\right)$; doubling time $\left(t_{\mathrm{D}}\right)$; ATP binding cassette (ABC). ly that this transporter contributes to its limited access to the brain. P-gp is expressed in high levels in cultured brain capillary endothelial cells and in intact brain capillaries $(15,16)$. It is localized at the luminal surface of the endothelium $(17,18)$, and therefore is in the correct location to restrict permeation of a variety of drugs into the CNS (19-21). Animals with reduced $\mathrm{p}$-gp function show an accumulation of p-gp substrates in the brain as well as a markedly increased sensitivity to neurotoxic $\mathrm{p}$-gp substrates, e.g., ivermectin $(18,22,23)$.

The present study identifies in vitro a mechanism that limits paclitaxel access to the CNS and outlines a strategy to circumvent it by blocking p-gp. We demonstrated that the p-gp blocker valspodar enhances paclitaxel entry into the brains of mice after intravenous dosing and that valspodar dramatically increases paclitaxel effectiveness against a human glioblastoma implanted into the CNS of nude mice. These are the first data directly demonstrating the role of $\mathrm{p}$-gp in limiting the therapeutic availability of paclitaxel to the CNS.

\section{Methods}

Chemicals. Valspodar (SDZ PSC 833) and the fluorescent cyclosporin derivative NBDL-CS were from Novartis Pharma GmbH (Nürnberg, Germany and Basel, Switzerland). Paclitaxel, BODIPY Fl-paclitaxel, 
and calcein-AM were from Molecular Probes Europe BV (Leiden, The Netherlands).

Capillary isolation. Capillaries from male Wistar rat brains were isolated at $4{ }^{\circ} \mathrm{C}$ in pregassed $\left(95 \% \mathrm{O}_{2} / 5 \%\right.$ $\mathrm{CO}_{2}$ ) solutions as described (18). Briefly, pieces of cortical gray matter were homogenized in three volumes (vol/wt) of buffer A (103 mM NaCl, $4.7 \mathrm{mM} \mathrm{KCl}, 2.5$ $\mathrm{mM} \mathrm{CaCl}_{2}, 1.2 \mathrm{mM} \mathrm{KH}_{2} \mathrm{PO}_{4}, 1.2 \mathrm{mM} \mathrm{MgSO}_{4}, 15 \mathrm{mM}$ HEPES), and, after addition of dextran (final concentration 30\%), the homogenate was centrifuged at low speed. The pellet was resuspended in buffer A supplemented with $25 \mathrm{mM} \mathrm{NaHCO}_{3}, 10 \mathrm{mM}$ glucose, $1 \mathrm{mM}$ Na-pyruvate, $0.5 \%$ (wt/vol) BSA, and filtered through a $200-\mu \mathrm{m}$ nylon mesh. The filtrate was passed over a glass bead column, and after washing, capillaries adhering to the beads were collected by gentle agitation. Capillaries were centrifuged, the pellet was resuspended in ice-cold, BSA-free Krebs-Henseleit buffer (KHB) and immediately used for transport experiments.

Fluorescence microscopy. Capillaries were transferred to a Teflon incubation chamber, containing $0.5-1.0 \mathrm{ml}$ of Krebs-Ringer medium with fluorescent compounds and transport effectors. Fluorescent compounds and inhibitors were added to the incubation medium in DMSO stock solutions. Earlier experiments showed that DMSO concentrations less than or equal to $0.5 \%$ had no significant effect on transport. The chamber floor was a $2 \times 2-\mathrm{cm}$ glass coverslip through which the tissue could be observed by a confocal laser scanning microscope (LSM 5 Pascal, 488-nm laser line, 515-nm long-pass emission filter; Carl Zeiss Jena GmbH, Jena, Germany). A low laser intensity ( $<3 \%$ of maximum) was used to avoid photobleaching. Tissue autofluorescence was not detectable. The images were analyzed with the Scion Image software (Beta 3; Scion Corp., Frederick, Maryland, USA). Epifluorescent images of capillaries were acquired by averaging four to eight frames. Earlier, using fluorescein as reference compound, it was shown that fluorescence intensity and fluorescein concentration had an approximately linear relationship (24). Here, the data are given as average of measured pixel intensity rather than estimated concentration of fluorescent compounds. Intensities were measured as described for a similar system of kidney proximal tubules (25). Several cellular and luminal areas were selected, and the average pixel intensity for each area was calculated. Analysis by high performance thin layer chromatography of BODIPY Fl-paclitaxel and NBDL-CS after extraction out of tissue with ethanol gave no hint for degradation or transformation of the fluorescent compounds for at least the time of incubation ( $\leq 1$ hour).

Isolation of endothelial cells and primary culture. Porcine brain capillary endothelial cells were isolated as described (26). Primary cultures were prepared according to ref. 27. Cells were collected and washed twice in medium containing $10 \%$ horse serum. The final suspension was kept in medium with $20 \%$ horse serum and $10 \%$ DMSO and stored in liquid nitrogen until use.
For uptake experiments, cells were seeded onto collagen-coated (Hoffman-LaRoche Ltd., Basel, Switzerland) 96-well plates. They were cultured under standard conditions using isolation medium without gentamycin, but with $10 \%$ horse serum. One day before transport experiments medium was replaced with $45 \%$ MEM, 45\% F12-HAM, $100 \mu \mathrm{g} / \mathrm{ml}$ streptomycin, 100 $\mu \mathrm{g} / \mathrm{ml}$ penicillin G, $10 \mathrm{mM}$ HEPES, $2 \mathrm{mM}$ glutamine, and $550 \mathrm{nM}$ hydrocortisone. For the calcein assay, cells were washed with KRB buffer $\left(37^{\circ} \mathrm{C}\right)$ and incubated with the test compounds for 5-15 minutes. CalceinAM $(1 \mu \mathrm{M})$ was added, and cells were incubated 15-45 minutes. Then they were washed three times with icecold KRB and lysed with $1 \%$ Triton X-100. Fluorescence was measured using a Fluoroskan Ascent reader (LabSystems GmbH, Frankfurt, Germany).

Western blots. P-gp was detected by Western blot analysis using the mAb C219 (Alexis Deutschland $\mathrm{GmbH}$, Grünberg, Germany). The p-gp-overexpressing cell line (MDR+)P388 was used as control. SDSPAGE was performed with a Mini-Protean II apparatus (Bio-Rad Laboratories GmbH, Munich, Germany). Proteins were transferred to nitrocellulose membranes using a Mini Trans-Blot cell for 2.5 hours at $250 \mathrm{~mA}$. The membranes were incubated with $\mathrm{Ab}$ (1:20 dilution) in PBS containing $0.05 \%$ Tween $20,1 \%$ BSA, and $1 \%$ milk powder for 2 hours at $37^{\circ} \mathrm{C}$. They were washed four times for 15 minutes and incubated 1 hour at room temperature with horseradish peroxidase-conjugated rabbit anti-mouse IgG (1:1000; Roche Molecular Biochemicals, Mannheim, Germany) in PBS containing $1 \%$ milk powder and $0.05 \%$ Tween 20. After washing, p-gp was detected using enhanced chemiluminescence (ECL) reagent (Amersham International, Buckinghamshire, United Kingdom).

Tumor cell lines and culture conditions. Human U-87 MG (HTB 14) and U-118 MG (HTB 15) glioblastoma/astrocytoma cell lines were obtained from American Type Culture Collection (Rockville, Maryland, USA). Cell banking and quality control were performed according to the "seed stock concept." U-87 MG cells were grown in Eagle's MEM containing L-glutamine, $2.2 \mathrm{~g} / 1 \mathrm{NaHCO}_{3}$, $110 \mathrm{mg} / \mathrm{l}$ sodium pyruvate, and $10 \%$ FCS. U-118 MG cells were maintained in DMEM supplemented with 10\% FCS. Cells were cultured in a carbogen atmosphere at $37^{\circ} \mathrm{C}$ and were passaged following treatment with trypsin $(0.05 \%) / \operatorname{EDTA}(0.02 \%)$. For tumor cell implantation into nude mice, cells were harvested mechanically, spun down, and washed twice with serum-free medium.

Chemosensitivity assay. The in vitro antitumor activity of paclitaxel and the modulating effect of valspodar were determined by a kinetic assay based on crystal violet staining. Processing procedure and data analysis were essentially performed as described (28). Paclitaxel and valspodar were added as 2,000-fold concentrated feed solutions in $70 \%$ ethanol. Doubling-time analysis of the growth curves of untreated controls was accomplished by a polynomial regression fit applying the least-squares method as earlier reported (29). 
Immunocytochemistry. Subconfluent tumor cells, cultured on chamber slides, were fixed with $4 \%$ phosphate buffered paraformaldehyde, $\mathrm{pH} 7.4$, for 30 minutes and washed three times with PBS. Endogenous peroxidase was blocked by incubation in PBS containing $3 \% \mathrm{H}_{2} \mathrm{O}_{2}$ and $10 \%$ methanol for 30 minutes, followed by two incubations in PBS for 5 minutes. Nonspecific binding was prevented by 30 -minute incubation in 10\% goat serum (Dianova, Hamburg, Germany), $2.5 \%$ skimmed milk powder (Milupa, Friedrichdorf, Germany), and $0.3 \%$ Triton X-100 in PBS. Subsequently, cells were washed three times with PBS containing 0.04\% Triton X-100 (PBST).

Primary Ab's AB-1 (PC03) (polyclonal antihuman/mouse p-gp, mdr; Dianova) and JSB-1/IgG-1 (monoclonal anti-human p-gp; Alexis Deutschland $\mathrm{GmbH}$ ) were diluted 1:50 with washing buffer consisting of $10 \mathrm{ml} \mathrm{PBST,} 100 \mu \mathrm{l} \mathrm{NGS}$, and $0.3 \%$ Triton $\mathrm{X}-100$. After incubation with primary Ab overnight at room temperature (RT), the slides were washed three times with PBS containing $0.34 \%$ Triton X-100 and treated with secondary $\mathrm{Ab}$ (for $\mathrm{Ab}-1$, biotinylated goat anti-rabbit, 1:200; Sigma-Aldrich, Deisenhofen, Germany; for JSB-1, biotinylated donkey anti-mouse 1:800; Dianova) for 2 hours at RT in a humid chamber. Then, cells were washed three times in PBST and incubated for 1 hour in horseradish-conjugated avidin-biotin complex (Vectastain Elite Kit ABC; Camon Labor Service GmbH, Wiesbaden, Germany) in PBST. Slides were washed three times for $10 \mathrm{~min}$ utes in PBST before incubation in a diaminobenzidine (DAB) solution for 10 minutes $(0.05 \% \mathrm{DAB}$, $0.02 \%$ ammonium nickel sulfate in PBST). $\mathrm{H}_{2} \mathrm{O}_{2}$ was added to a final concentration of $0.006 \%$, and slides were incubated for 10 minutes. Subsequently, they were washed three times in PBS, processed in an ascending alcohol series, and mounted in DePex.

Immunohistochemistry. Isolated brain capillaries were transferred onto slides and fixed for 20 minutes with $3 \%$ paraformaldehyde, $0.1 \%$ glutardialdehyde, and $3.4 \%$ sucrose in PBS. Capillaries were permeabilized for 15 minutes with $1 \%$ (vol/vol) Triton X-100 in PBS. Then, the capillaries were incubated for 1 hour at $37^{\circ} \mathrm{C}$ in a humid chamber with primary $\mathrm{Ab}(150 \mu \mathrm{l}$ murine $\mathrm{mAbB} C 219,1: 20$ dilution). After washing the corresponding fluorochrome-conjugated secondary $\mathrm{Ab}(150 \mu 1$ FITC-conjugated rabbit anti-mouse IgG, 1:20 dilution; Hoffmann-LaRoche Ltd.) was added for 1 hour in a humid chamber in the dark.

Murine tissues and intracerebrally growing human tumors were fixed in Bouin's solution for 3 days, then were dehydrated and embedded into paraffin. Sections of $5-\mu \mathrm{m}$ thickness were prepared, deparaffinated with xylene, and rehydrated in a descending alcohol series. P-gp was detected with the polyclonal Ab-1 (PC03) Ab (anti-human and murine p-gp; Dianova) with nuclear fast-red counter staining. The protocol was the same as for immunocytochemistry except that the primary $\mathrm{Ab}$ was diluted $1: 10$ with washing buffer.
Pharmacokinetic studies in nude mice. NMRI nu/nu mice were bred under specified pathogen-free conditions at $26^{\circ} \mathrm{C}, 70 \%$ relative humidity, and a 12 -hour light/ 12 hour dark cycle at the University of Regensburg (30). They were fed ad libitum with a breeding/maintenance diet (Altromin GmbH, Lage, Germany) and water containing $1.3 \mathrm{~g} / \mathrm{l}$ potassium sorbate and $2 \mathrm{~g} / \mathrm{l}$ chloramphenicol; $\mathrm{pH}$ was adjusted to 2.5 with $\mathrm{HCl}$. The use of animals in this study complies with the Guide for the Care and Use of Laboratory Animals (NIH publication no. 86-23, revised 1985) and the current German law on the protection of animals.

Prior to drug administration, 6- to 8-week-old female NMRI $(n u / n u)$ mice were assigned to groups of three to four animals per time point. Drinking solution containing $100 \mathrm{mg} / \mathrm{ml}$ valspodar was diluted to a concentration of $5 \mathrm{mg} / \mathrm{ml}$ with its vehicle, a mixture of Cremophor RH40, ethanol, D,L- $\alpha$-tocopherol, propylene glycol, and Labrafil M2125CS. A volume of $0.2 \mathrm{ml}$ was administered by gavage per 20 $\mathrm{g}$ of body weight, resulting in a dosage of $50 \mathrm{mg}$ valspodar per kilogram of body weight. In the same way, the solvent was applied to the control animals.

For intravenous injection the commercial drug formulation Taxol $(6 \mathrm{mg} / \mathrm{ml}$ paclitaxel; Bristol-Myers Squibb) was diluted with $0.9 \% \mathrm{NaCl}$ to a paclitaxel concentration of 1.6 or $0.8 \mathrm{mg} / \mathrm{ml}$. The drug was injected via the retrobulbar plexus in a volume of $0.1 \mathrm{ml}$ per 20 g of body weight at a dosage of 8 or $4 \mathrm{mg}$ paclitaxel per $\mathrm{kg}$ body weight within approximately 10 seconds. Four hours before the injection of paclitaxal, either a dose of $50 \mathrm{mg} / \mathrm{kg}$ valspodar (valspodar group) or a corresponding volume of the vehicle of valspodar (control) was administered to the mice by mouth.

To minimize adulterations of the analytical results by drug in the vasculature, ketamine/xylazine-anesthetized mice were killed by cardiac puncture, 1.5, 3 , and 24 hours after intravenous injection, and heparinplasma, brain, liver, and kidneys were collected. Tissues were homogenized in 4\% BSA within 15 minutes and shock-frozen in liquid nitrogen. For homogenization, each organ was minced into small pieces. One milliliter 4\% BSA solution was added per $150 \mathrm{mg}$ tissue, and homogenization was carried out with a Potter-Elvehjem homogenizer by 15 strokes. All samples were stored at $-78^{\circ} \mathrm{C}$.

Paclitaxel analysis. Tissue extraction was by a modification of the method of Sparreboom et al. (31). Thawed tissue homogenate $(1-2 \mathrm{ml})$ was pipetted into a glass centrifugation tube with a ground joint. Samples less than $1 \mathrm{ml}$ were filled up to $1 \mathrm{ml}$ with $4 \%$ BSA solution. Tissue samples underwent diethyl ether extraction and solid-phase extraction (SPE). Plasma samples consisted of $250 \mu \mathrm{l}$ of thawed murine plasma. Samples with less than $250 \mu \mathrm{l}$ volume were filled up to $250 \mu \mathrm{l}$ with human citrate-phosphate-dextrose/plasma. Only plasma samples underwent SPE.

To each sample $10 \mu \mathrm{l}$ of $50 \mu \mathrm{M}$ docetaxel solution was added as internal standard. For docetaxel stock 
solutions Taxotere $(80 \mathrm{mg}$ docetaxel per $2 \mathrm{ml}$ of polysorbate 80; Rhone-Poulenc Rorer, Antony Cedex, France) was diluted with absolute ethanol. HPLC analysis was performed on a Thermo Separation Products liquid chromatograph (Thermo Quest; Egelsbach, Germany). A stainless steel $(125 \times 4 \mathrm{~mm})$ analytical column equipped with a guard column $(4 \times 4 \mathrm{~mm})$, both packed with $5 \mu \mathrm{m}$ LiChrospher $100 \mathrm{RP}-18$ material (Merck kGaA, SLP PLS, Darmstadt, Germany), was cooled to $33^{\circ} \mathrm{C}$. The mobile phase consisted of acetonitrile/methanol $/ 0.2 \mathrm{M}$ ammonium acetate buffer, $\mathrm{pH}$ 5.0, 38:10.5:51.5 (vol/vol). Ultraviolet detection was performed at $227 \mathrm{~nm}$, and a washing gradient was applied after each run (additional 35\% vol/vol acetonitrile). Injection volumes were either 50 or $100 \mu \mathrm{l}$. For calibration, paclitaxel and docetaxel stock solutions in anhydrous ethanol, stored at $-20^{\circ} \mathrm{C}$, were diluted with mobile phase (1:100) on the day of analysis.

The method of analysis was validated for murine plasma, brain, liver, and kidney samples (32). Correlation coefficients for paclitaxel calibration curves in the concentration range $50-1,000 \mathrm{nM}$ were greater than 0.999 . Recovery rates for paclitaxel ranged from 50 to $85 \%$, depending on paclitaxel concentration and the type of tissue. The method's accuracy for tissue samples was below $2 \%$ for paclitaxel concentrations between 0.667 and $6.67 \mathrm{nmol} / \mathrm{g}$ of tissue and better than $8 \%$ for $0.133 \mathrm{nmol} / \mathrm{g}$. The precision for these determinations was better than $10 \%$.

Tumor implantation, treatment, and evaluation. In the intracerebral model, the parietal bone of anaesthetized male nude mice (mean body weight $35 \mathrm{~g}$ ) was drilled though with a $1-\mathrm{mm}$-diameter bit $3 \mathrm{~mm}$ on the righthand side of the sagittal line and $3 \mathrm{~mm}$ rostral of the coronal line. Human U-87 MG cells $\left(10^{6}\right.$; glioblastoma/astrocytoma grade III) or 300,000 U-118 MG (glioblastoma) tumor cells suspended in $10 \mu \mathrm{l}$ of serum-free Eagle's minimum essential medium were injected $3 \mathrm{~mm}$ deep into the brain tissue, and the wound was closed with a surgical clamp.

Mice were assigned to three groups $(n=7)$. The control group received no medication. Two additional groups were treated as described for the pharmacokinetic experiment either with a combination of valspodar and paclitaxel or the vehicle of valspodar and paclitaxel. Valspodar $(50 \mathrm{mg} / \mathrm{kg})$ and vehicle were given to the animals inoculated with the U-87 MG tumors by mouth 4 hours before the intravenous injection of two doses of paclitaxel, i.e., $4 \mathrm{mg} / \mathrm{kg}$ on day 7 and another $2 \mathrm{mg} / \mathrm{kg}$ on day 14 after tumor implantation. Animals were killed at day 20 , and brains were fixed in Bouin's solution for histological examination. Animals bearing intracerebral U-118 MG tumors were treated analogously, except drugs were administered on days 8 and 15 , the first paclitaxel dose was reduced to $3 \mathrm{mg} / \mathrm{kg}$, and brains were removed for morphometric analysis on day 35 .

Brains were fixed in Bouin's solution for 3 days, dehydrated, and embedded into paraffin. Serial 5- $\mu \mathrm{m}$ coronal sections were prepared every $200 \mu \mathrm{m}$ (U-87
MG) or $300 \mu \mathrm{m}$ (U-118 MG) starting and ending 1.5 $\mathrm{mm}$ rostral and caudal to the region of tumor cell implantation, deparaffinated, and stained according to Masson and Goldner (33). Tumor areas on nine central sections were determined per brain morphometrically with the software Metamorph (Visitron Systems GmbH, Puchheim, Germany), and the tumor volume in the investigated brain region was calculated.

In the subcutaneous model, approximately $5 \times 10^{6}$ U-118 MG cells $(100 \mu \mathrm{l})$ were injected into the right flank of 6-week-old female NMRI $(n u / n u)$ mice. Solid tumors developed within 8 weeks. For serial passage, the tumors were cut into $2-\mathrm{mm}^{3}$ pieces and transplanted subcutaneously into the thoracic region with a trocar (30).

Mice were assigned to four groups of ten animals. The control group received no medication, another group received the complex vehicle of valspodar, whereas valspodar was administered to the third and fourth groups at a dosage of 10 and $50 \mathrm{mg} / \mathrm{kg}$, respectively. Valspodar and its vehicle were administered orally twice per week, resulting in cumulative doses of 120 and $600 \mathrm{mg}$ valspodar per kilogram. On day 46 after tumor transplantation the animals were killed by cervical dislocation.

Tumor growth kinetics was recorded by measuring tumor diameters with an electronic caliper. Tumor areas were calculated as the product of two perpendicular diameters, of which one represents the largest possible diameter of the tumor. Tumor size and the weight of the animals were measured twice a week.

Statistical analysis. Data are presented as mean values plus or minus SEM and were evaluated by one-way ANOVA using SPSS 10.0 for Windows. Except for the pharmacokinetic results, which were analysed nonparametrically by the $U$ test according to Mann and Whitney, the unpaired Student $t$ test for inhomogeneous variances was applied. $P$ values less than or equal to 0.05 were considered statistically significant.

\section{Results}

Isolated brain capillaries. Integrity of isolated capillaries was checked by microscopy. After a 60- to 80minute incubation with FITC-dextran 4000 as a marker for paracellular permeation, no fluorescence was detected inside the capillary lumina by confocal scanning microscopy, indicating that tight junction integrity was maintained and that the endothelium of isolated capillaries is a significant barrier to diffusion of large molecules, which is consistent with in vivo observations.

Immunohistochemical assessment of p-gp in intact rat capillaries (Figure 1a) showed the primary localization of the export protein in the luminal membrane, with only weak staining at the outer membrane. Presence of p-gp was confirmed by Western blots of porcine brain capillary endothelial cells that were cultured for 7 days prior to transport studies (Figure 1b). In the human brain, p-gp (MDR1) was found in capillaries in the cortex, the hippocampus, the cerebellum, and the spinal cord, but not in submeningeal blood 


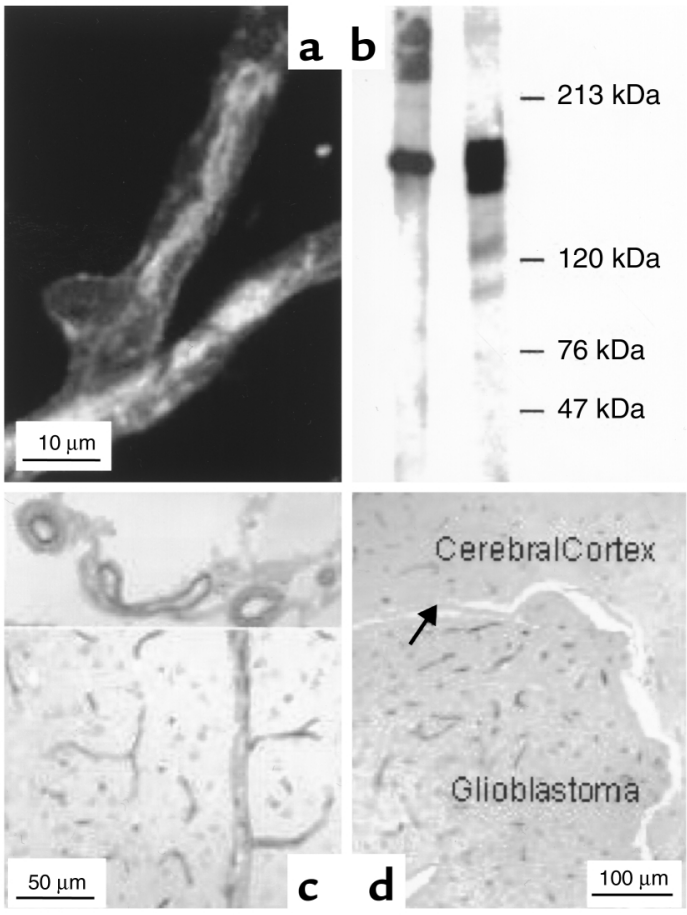

vessels and the vessels of the choroid plexus (34). In coronal sections of the brains of NMRI nude mice, $\mathrm{p}$ pg (AB-1 Ab's) was not only detected in capillaries of the cortex but also in deeper medullar regions. Figure $1 \mathrm{c}$ shows that not only small capillaries and larger blood vessels in the cortex stained positive, but also that $\mathrm{p}$-gp is expressed in submeningeal arteries and veins. The immunopositive small capillaries were unevenly distributed in the cerebral parenchyma, reflected in a somewhat lower density between the outer band of Baillarger and Kaes-Bechterew's band (results not shown). Hepatic bile canaliculi, renal proximal tubules, and small intestinal brush borders also stained positive (not shown).

\section{Figure 1}

(a) Immunostaining of $\mathrm{p}$-gp in a freshly isolated rat brain capillary (primary Ab: MAB C219; secondary Ab: FITC-conjugated rabbit antimouse IgG). (b) P-gp detection by Western blot using the MAB C219. Left lane: brain capillaries; right lane: $p$-gp overexpressing cell line (MDR+)P388 as positive control. (c) Immunostaining of coronar sections of the brains of nude mice, using the polyclonal $A B-1$ Ab. Healthy animal, with P-gp expression in capillaries and larger blood vessels in the cerebrum (bottom) and the submeningeal arteries and veins (top). (d) Intracerebral human glioblastoma (U-87 MG) with immunopositive capillary endothelia and negative tumor cells. The arrow points to the ependyma.

Figure 2a shows the time course of BODIPY Fl-paclitaxel accumulation in intact rat brain capillaries. Fluorescence in cells and capillary lumina rapidly increased, reaching steady-state values after 30-45 minutes. At steady state, luminal fluorescence was about three times cellular fluorescence. Medium fluorescence was barely detectable, so BODIPY Fl-paclitaxel clearly accumulated in the cells and lumina. In capillaries exposed to $1 \mathrm{mM} \mathrm{NaCN}$, steady-state luminal fluorescence was significantly reduced compared with controls, but cellular fluorescence was unchanged (Figure 2a). With $\mathrm{NaCN}$, luminal fluorescence approached cellular levels. Thus, accumulation of BODIPY Fl-paclitaxel within the cells was not energy dependent, probably reflecting passive diffusion, binding to cellular elements, and partitioning into membranes. In contrast, movement of BODIPY Fl-paclitaxel from cell to lumen was energy dependent, suggesting uphill transport. Luminal accumulation of BODIPY Fl-paclitaxel was reduced in a concentration-dependent manner by unlabeled paclitaxel, suggesting that both compounds interact with the same transporter. Luminal accumulation of BODIPY Fl-paclitaxel was also reduced by the p-gp inhibitor, valspodar (Figure 2b). Neither paclitaxel nor valspodar reduced cellular fluorescence. In contrast, the Mrp
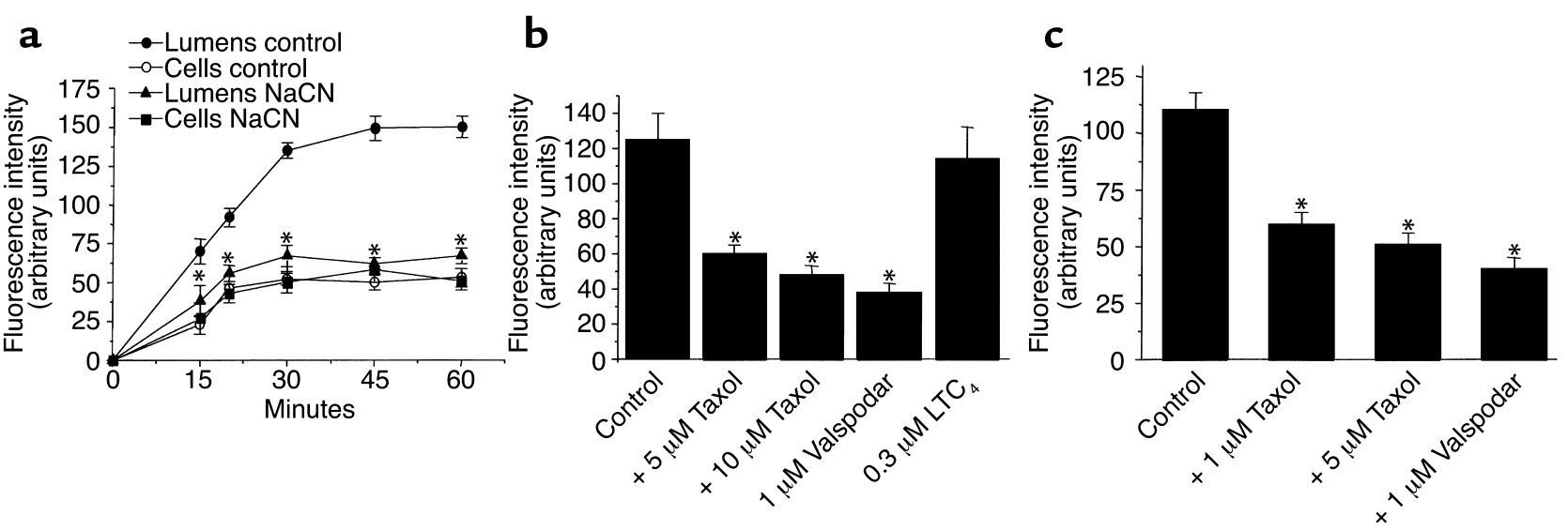

Figure 2

(a) Time-dependent accumulation of BODIPY Fl-paclitaxel in cells and lumens of isolated rat brain capillaries in the absence and presence of $1 \mathrm{mM} \mathrm{NaCN}$. Means $\pm \mathrm{SEM}, n=10-12, P=0.05$. (b) Accumulation of BODIPY FI-paclitaxel and NBDL-CS (c), respectively, in the lumens of isolated capillaries in the absence and presence of unlabeled paclitaxel, the p-gp blocker valspodar, and the mrp2 substrate LTC 4 . The fluorescent compounds were added at concentrations of $1 \mu \mathrm{M}$. Means $\pm \mathrm{SEM}, n=10-12$. ${ }^{*}$ Significantly different from controls; $P=0.05$. 


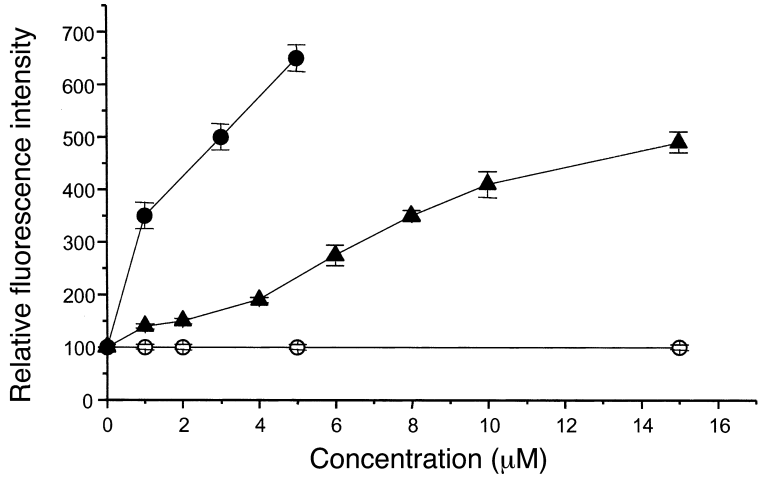

Figure 3

Increase of intracellular fluorescence intensity (free calcein) in monolayers of porcine brain capillary endothelial cells. Presence of the p-gp substrates valspodar (filled circles) and paclitaxel (filled triangles) results in an increased fluorescence intensity, whereas penicillin (open circles) has no effect (means $\pm \mathrm{SEM}, n=6$ ).

inhibitor leukotriene $\mathrm{C}_{4}\left(\mathrm{LTC}_{4}\right)$, was without effect. We also examined the effects of paclitaxel on the transport of NBDL-CS, a fluorescent p-gp substrate $(25,35)$. As with BODIPY Fl-paclitaxel, luminal accumulation of NBDL-CS was inhibited by valspodar and by paclitaxel (Figure 2c). Cellular fluorescence was not affected by valspodar or paclitaxel (not shown).

Calcein-AM assay in primary cultures of porcine brain capillary endothelial cells. We confirmed the above results using a fluorescence-based $\mathrm{p}$-gp assay and monolayer cultures of porcine brain capillary endothelial cells expressing $\mathrm{p}$-gp. In this assay calcein-AM is excluded from the cells by p-gp. When p-gp is inhibited, e.g., by competitors, the compound accumulates intracellularly and is hydrolyzed to calcein. This fluorescent organic anion is trapped within the cells, and the accumulation is taken as a measure of transporter inhibition. Under control conditions, calcein accumulation was very low. However, when valspodar or paclitaxel was added, concentration-dependent increases in calcein accumulation were observed. Although paclitaxel was not quite as potent an inhibitor of $\mathrm{p}$-gp as valspodar, it was also effective in the $\mu \mathrm{M}$ concentration range (Figure 3 ).

Pharmacokinetic studies in nude mice. In mice given 8 $\mathrm{mg} / \mathrm{kg}$ paclitaxel intravenously, concentrations in plas- ma averaged $1.45 \pm 0.28 \mu \mathrm{M}$ and $0.48 \pm 0.04 \mu \mathrm{M} 1.5$ and 3 hours after dosing, respectively. Pretreatment with valspodar $(50 \mathrm{mg} / \mathrm{kg}$ by mouth) slightly increased plasma concentration at 1.5 hours $(2.51 \pm 0.64 \mu \mathrm{M})$ and significantly increased plasma concentration after 3 hours $(3.22 \pm 0.20 \mu \mathrm{M})$. For both groups, plasma paclitaxel concentrations were below the limit of detection 24 hours after injection.

In mice that received paclitaxel alone, brain levels of the drug were always near the limit of detection. Coadministration of valspodar increased the paclitaxel levels in the brain by about one order of magnitude. In contrast to plasma, liver, and kidney (Table 1) this elevation in brain paclitaxel was rapid and long lasting; i.e., the levels were elevated after 1.5 hours and remained high for at least 24 hours after dosing (see Figure 5).

In clinical trials, paclitaxel doses had to be reduced by $30-60 \%$ if valspodar was coadministered $(36,37)$. Therefore, paclitaxel tissue concentrations were determined in mice that were pretreated with valspodar but received half the paclitaxel dose $(4 \mathrm{mg} / \mathrm{kg}$; Figure 4). Despite the reduced dose, 24 hours after injection paclitaxel levels were three times higher in the brains of these pretreated mice than in animals that had received $8 \mathrm{mg}$ paclitaxel/kg without valspodar (Figure 5). Paclitaxel levels in liver and kidney of the animals in the PSC 833 group with the reduced paclitaxel dose were roughly comparable to the PSC 833 group with the full paclitaxel dose.

Characterization of the human glioblastoma cell lines. When diagnosed, some neuroepithelial tumors, especially astrocytoma and neuroblastoma, exhibit the classical primary MDR phenotype (38-40). Moreover, in astrocytoma, $\mathrm{p}$-gp expression seems to be positively correlated with tumor grade (41). Therefore, the human U-87 MG and the U-118 MG glioblastoma/ astrocytoma cells were checked for p-gp expression using the monoclonal JSB-1 Ab, which recognizes an uncharacterized epitope on the cytoplasmic side of the human MDR1 p-gp (42), and using the polyclonal Ab $\mathrm{AB}-1$, which binds to a cytoplasmic domain at the $\mathrm{C}$ terminus of human MDR1 p-gp and murine mdr1a and $\mathrm{mdr} 1 \mathrm{~b}$ with similar affinity. P-gp-overexpressing KB-V1 cells, cultured in the presence of $300 \mathrm{ng} / \mathrm{ml}$ vinblastine, were used as positive controls. Whereas

Table 1

Effect of valspodar coadministration on paclitaxel levels (mean values \pm SEM) in liver and kidney of nude mice $(n=3)$ after intravenous injection of $8 \mathrm{mg} / \mathrm{kg}$ paclitaxel

\begin{tabular}{|c|c|c|c|c|}
\hline \multirow[b]{3}{*}{ Time (h) } & \multicolumn{3}{|c|}{ Mean paclitaxel level \pm SEM $(\mathrm{nmol} / \mathrm{g})$} & \\
\hline & & & \multicolumn{2}{|c|}{ Kidney } \\
\hline & Paclitaxel & Valspodar plus paclitaxel & Paclitaxel & Valspodar plus paclitaxe \\
\hline 1.5 & $33.49 \pm 1.87$ & $18.50 \pm 1.18^{\mathrm{A}}$ & $12.25 \pm 0.63$ & $15.66 \pm 0.46^{\mathrm{A}}$ \\
\hline 3 & $18.03 \pm 2.98$ & $20.17 \pm 0.74$ & $7.87 \pm 0.65$ & $17.87 \pm 0.66^{\mathrm{A}}$ \\
\hline 24 & $0.91 \pm 0.24$ & $2.28 \pm 0.34^{\mathrm{A}}$ & $0.37 \pm 0.24$ & $2.62 \pm 0.69^{A}$ \\
\hline
\end{tabular}

Four hours before intravenous application the animals were treated with the vehicle of valspodar by mouth (paclitaxel) or $50 \mathrm{mg} / \mathrm{kg}$ valspodar by mouth (valspodar plus paclitaxel). AStatistically significant differences $(P \leq 0.05)$. 
Control group

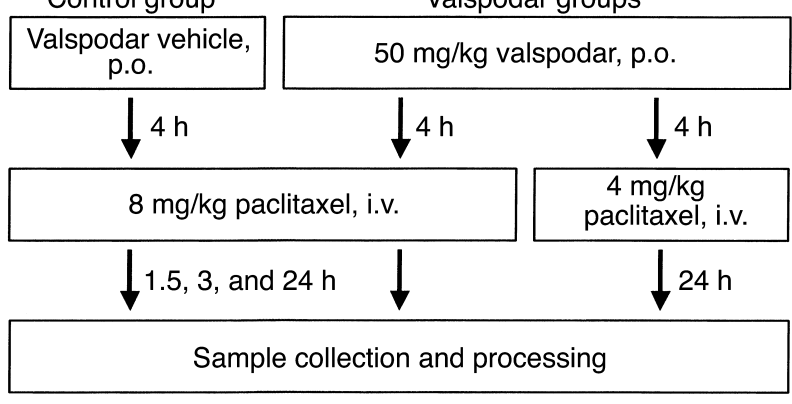

Figure 4

Application scheme of PSC 833 and paclitaxel to nude mice. Each group consisted of three to four nude mice per observation time after intravenous injection.

KB-V1 cells stained intensely with both Ab's, U-87 MG and U-118 MG glioma cells did not.

When inoculated intracerebrally, the human glioblastoma cell lines U-87 MG and U-118 MG formed solid tumors as shown in the Figure $1 \mathrm{~d}$. Immunostaining with the $\mathrm{AB}-1 \mathrm{Ab}$ revealed $\mathrm{p}-\mathrm{gp}$ expression in the neovasculature, which is consistent with the clinical situation (43-45). On the contrary, in agreement with the results of immunocytochemistry the tumor cells did not exhibit the MDR1 phenotype and did not stain with the monoclonal anti-human p-gp 170 JSB-1 Ab (not shown).

Within the tumor, blood vessels of varying diameters were immunopositive. Staining intensity of the tumor endothelia depended on tumor size and localization. Highest p-pg expression was observed in small neoplasms, especially in the proliferating peripheral region. In this area, density and staining intensity of the newly formed capillaries were higher than of the blood vessels in the surrounding brain parenchyma. In contrast, when the glioma was implanted into nude mice subcutaneously, the tumor vessels failed to express p-gp (46).

To determine the sensitivity of U-87 MG and U-118 MG glioblastoma cells against paclitaxel and valspodar, the cells were exposed to the drugs during the whole incubation period of up to 300 hours. Doubling times substantially differed between the two cell lines. For U-87 MG a mean minimal doubling time $\left(t_{\mathrm{D}}\right)$ of 27.5 hours was determined, whereas proliferation of U-118 MG cells with a $t_{\mathrm{D}}$ of 50 hours was significantly slower (47). At a concentration of $100 \mathrm{nM}$ (a typical peak plasma concentration), paclitaxel was cytostatic on U-87 MG cells, whereas proliferation of the U-118 MG cells had already ceased at $5 \mathrm{nM}$. At a concentration of $1 \mu \mathrm{M}$, valspodar did not affect proliferation of either cell line. Combining $1 \mu \mathrm{M}$ valspodar with weakly cytotoxic concentrations of doxorubicin, etoposide, and paclitaxel, known substrates of p-gp 170, failed to enhance the cytotoxicity of these p-gp substrates. Therefore, both cell lines appear to be well suited to investigate the effect of valspodar paclitaxel coapplication at the blood-brain barrier.
Paclitaxel effects on intracerebral growth of human glioma. Two experiments were carried out to determine whether the rise in the brain levels of paclitaxel in animals pretreated with valspodar could provide a therapeutic benefit. In the first experiment, human U-87 MG glioblastoma cells were implanted intracerebrally into nude mice. This procedure results in aggressively growing intracerebral neoplasms (48). As described above, this cell line is relatively insensitive to paclitaxel and certain other chemotherapeutic agents in vitro. The animals $(n=7-8)$ were dosed with $4 \mathrm{mg} / \mathrm{kg}$ paclitaxel on day 7 after implantation and with $2 \mathrm{mg} / \mathrm{kg}$ paclitaxel on day 14 , without and with $50 \mathrm{mg} / \mathrm{kg}$ valspodar 4 hours before each paclitaxel dose. Brains were collected on day 20 , and the intracerebral tumor tissue was quantified by morphometry. In this experiment, there were no statistically significant differences in the mean tumor volumes within the three groups: $7.4 \pm 1.3 \mathrm{~mm}^{3}$ in the control, $8.6 \pm 1.2 \mathrm{~mm}^{3}$ in the paclitaxel, and $7.8 \pm 1.3 \mathrm{~mm}^{3}$ in the paclitaxel-valspodar group. Whereas the mean body weight of the control animals increased by $5 \%$, there was a significant reduction of mean body weight in both treatment groups with a decrease by $9 \%$ in the paclitaxel group and a weight loss by $17 \%$ in the paclitaxel-valspodar group during the course of the experiment.

In the second study with intracerebral U-118 MG tumors, paclitaxel dosage was reduced to avoid weight loss. A control group received no medication; a second group received paclitaxel 8 days $(3 \mathrm{mg} / \mathrm{kg})$ and 15 days $(2 \mathrm{mg} / \mathrm{kg})$ after tumor implantation; a third group received the same regimen of paclitaxel dosing, but in combination with $50 \mathrm{mg} / \mathrm{kg}$ valspodar 4 hours before each paclitaxel dose. Mice were killed 35 days after

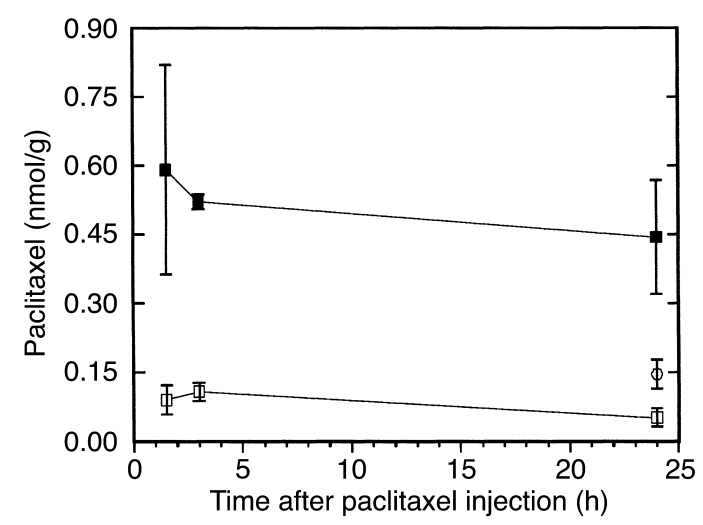

\section{Figure 5}

Effect of valspodar coadministration on the paclitaxel brain levels in nude mice after intravenous injection of $8 \mathrm{mg} / \mathrm{kg}$ paclitaxel. Four hours before intravenous application the animals were treated with the vehicle of valspodar by mouth (control, open squares) or 50 $\mathrm{mg} / \mathrm{kg}$ valspodar by mouth (valspodar group, filled squares). Brain levels for a valspodar group with reduced paclitaxel dose $(4 \mathrm{mg} / \mathrm{kg}$, open circles) were investigated 24 hours after intravenous injection. Means $\pm \mathrm{SEM}, n=3-4$. Compared with the respective controls, all values in the valspodar groups were significantly $(P \leq 0.05)$ higher. 


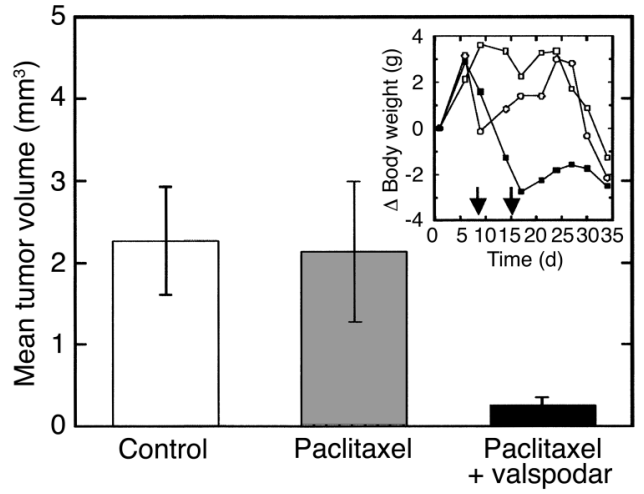

Figure 6

Effect of valspodar/paclitaxel coadministration on the intracerebral growth of the human $\mathrm{U} 118 \mathrm{MG}$ glioblastoma in nude mice. Controls received no medication, the paclitaxel group received the vehicle of valspodar 4 hours before injection of paclitaxel, and the paclitaxel-valspodar group received $50 \mathrm{mg} / \mathrm{kg}$ valspodar by mouth before injection of paclitaxel. Animals were treated in this way on day 8 ( 3 $\mathrm{mg} / \mathrm{kg}$ paclitaxel) and day $15(2 \mathrm{mg} / \mathrm{kg}$ paclitaxel) after implantation of tumors. Thirty-five days after tumor implantation the brains of the animals were collected and the tumor volumes determined morphometrically. The figure shows mean values $\pm \operatorname{SEM}(n=5)$. Inset: Change of mean body weight as an indicator of toxicity. Control, open squares; paclitaxel, open circles; paclitaxel-valspodar, filled squares. The arrows indicate drug administration.

implantation, and the tumor volume in each brain was determined morphometrically $(n=5)$. Treatment with paclitaxel alone did not reduce the tumor volume significantly, whereas valspodar plus paclitaxel decreased the tumor volume by $90 \%(P<0.05$; Figure 6$)$. As shown in Figure 6 (inset), the regimen was not compromised by intolerable toxicity. This result demonstrates that coadministration of valspodar can improve the therapeutic benefit of paclitaxel for brain tumor treatment. Clearly, in vivo the solid U-118 MG tumors were more sensitive to paclitaxel than the solid U-87 MG tumors, a result that agrees with proliferation kinetics and drug sensitivity of these cell lines in vitro $(46,47)$.

Effect of valspodar on subcutaneous growth of implanted buman glioma. Recently, valspodar was reexamined as an antitumor agent in vitro (49). We considered the possible contribution of valspodar to glioma cytotoxicity in an experiment with nude mice bearing U-118 MG glioblastoma subcutaneously. In a 46-day experiment, mice with implanted tumors were given intravenously 120 or $600 \mathrm{mg} / \mathrm{kg}$ valspodar, vehicle alone, or no treatment. At the end of the experiment, there were no statistically significant effects on tumor growth among the untreated control group, the vehicle group, and the two valspodar groups (Table 2), nor was there a hint of systemic or CNS toxicity.

\section{Discussion}

Malignant brain tumors (higher-grade gliomas) are rarely cured by surgery and/or radiotherapy, and chemotherapy has been of limited value, especially as long as the blood-brain barrier remains intact. In addition, the brain is sanctuary for metastases in cancer patients otherwise responding to cytostatic drugs such as paclitaxel (50).

One aim of this study was to find a mechanistic explanation for the low permeability of paclitaxel using in vitro and in vivo studies. Since paclitaxel is excluded from many tumor cells by p-gp, it is likely that this drug efflux pump also limits the access of the drug to the brain. Brain capillaries express p-gp and other ATP binding cassette (ABC) transporters at their luminal membrane $(16,51)$. To study the impact of $\mathrm{ABC}$ transporters on paclitaxel transport we applied a newly developed approach using isolated rat brain capillaries, fluorescent substrates, and confocal imaging. Isolated rat brain capillaries accumulated fluorescently labeled paclitaxel within the lumina. This accumulation was up to 3.5 -fold above the cellular accumulation and showed all the hallmarks of specific active transport. It was blocked when metabolism was inhibited by $\mathrm{NaCN}$ and when unlabeled drug or the p-gp modifier, valspodar, were added. Unlabeled paclitaxel inhibited the secretion of NBDL-cyclosporin, a p-gp substrate. In cultured endothelial cells from porcine brain, paclitaxel enhanced in a dose-dependent manner the intracellular accumulation of calcein derived from calcein-AM, a p-gp substrate that is otherwise excluded from the cells. All results point to $\mathrm{p}$-gp as a major obstacle to paclitaxel entry into the CNS.

Valspodar is a potent inhibitor of $\mathrm{p}$-gp that alters drug transport across barrier and excretory tissues in vitro and in vivo. As a result, it has the potential to alter the pharmacokinetics of the many therapeutic agents

Table 2

Effect of valspodar on the growth of subcutaneously implanted U-118 MG glioma

\begin{tabular}{|c|c|c|c|c|c|}
\hline \multirow{3}{*}{ Treatment group $(n=10)$} & \multicolumn{5}{|c|}{ Mean tumor area $\pm \operatorname{SEM}\left(\mathrm{mm}^{2}\right)$} \\
\hline & & & Day & & \\
\hline & 0 & 11 & 25 & 36 & 46 \\
\hline Control & $3.5 \pm 0.47$ & $6.64 \pm 0.82$ & $20.2 \pm 4.06$ & $24.5 \pm 5.62$ & $36.5 \pm 10.05$ \\
\hline $10 \mathrm{mg} / \mathrm{kg}$ valspodar & $2.9 \pm 0.48$ & $9.1 \pm 1.22$ & $18.0 \pm 3.60$ & $41.0 \pm 9.96$ & $53.7 \pm 27.80$ \\
\hline$P$ & $(0.423)$ & $(0.104)$ & $(0.699)$ & $(0.141)$ & $(0.550)$ \\
\hline $50 \mathrm{mg} / \mathrm{kg}$ valspodar & $3.4 \pm 0.43$ & $7.2 \pm 0.88$ & $18.3 \pm 2.53$ & $28.3 \pm 4.48$ & $35.2 \pm 12.94$ \\
\hline$P$ & $(0.933)$ & $(0.645)$ & $(0.719)$ & $(0.608)$ & $(0.948)$ \\
\hline
\end{tabular}

Valspodar was administered orally twice per week resulting in cumulative doses of 120 and 600 mg valspodar per kilogram. Treatment was started on day 1.

ASignificance level with respect to the control. 
that are p-gp substrates, leading to longer terminal half lives and lower excretion rates. Such effects have been observed for paclitaxel and other P-gp substrates in clinical and preclinical investigations $(23,36,37)$.

Other groups have shown that transporter ablation/inhibition increases drug delivery to the CNS. For example, van Asperen et al. (52) observed the tissue distribution of vinblastine in mice with homozygously disrupted mdrla genes (mdrla encodes for P-gp in mice predominantly expressed at the blood-brain barrier) and in the wild-type species. As in our work, they found the highest increase in tissue levels of mdr1a-deficient mice compared with the wild-type species for the brain, where 7-46 times higher vinblastine levels were found, depending on time and dose. Also consistent with our results, vinblastine levels in liver and kidney were significantly higher in knockout mice compared with wildtype animals no earlier than 24 hours after drug administration. The area under the plasma-time curve was found to be 1.4-1.6 times higher in mdr1a-deficient mice. Our results agree in principle with an increased plasma area under the curve. However, this fact cannot explain the six- to eightfold higher paclitaxel concentrations in the brain of valspodar pretreated animals within the first observation period of 1.5 hours, since the plasma levels were only slightly elevated compared with paclitaxel application alone. This supports the interpretation that the inhibition of p-gp by valspodar reduces paclitaxel efflux from the brain. With the available analytical techniques it is impossible to determine whether the drug is homogeneously distributed or accumulated by endothelial or other cells.

Drion et al. (53) perfused brains of rats for 20 seconds with radiolabeled vinblastine and analyzed brain sections. For rats that received $10 \mathrm{mg} / \mathrm{kg}$ valspodar intravenously 5 minutes before infusion, nine times higher vinblastine levels were found in all sections of gray brain tissue compared with rats that received no valspodar. These experiments are especially interesting, because the administration of valspodar has no influence on drug concentration in the perfusate and the influence of valspodar on the pharmacokinetics of the cytostatic is of no relevance.

The present results also show that valspodar pretreatment not only increased brain levels of paclitaxel, but that it also produced a therapeutic effect on a paclitaxel-sensitive transplanted tumor. Although paclitaxel therapy by itself did not affect the volume of intracerebrally implanted U-118 MG tumors, combined valspodar-paclitaxel therapy resulted in a significant decrease in tumor volume (Figure 6). In contrast, neither paclitaxel nor valspodar-paclitaxel therapy affected the volume of implanted U-87 MG tumors derived from a cell line that is not paclitaxel sensitive. Neither glioblastoma cell line exhibited a multidrug-resistance phenotype. Thus, the statistically significant reduction in tumor volume caused by valspodar coadministration was due to increased paclitaxel levels in the brain, a result of inhibition of P-gp-mediated transport at the blood-brain barrier. Circumvention of multidrug resistance at the tumor cell was not a factor. These findings suggest that coadministration of valspodar can be of clinical benefit for the chemotherapeutical treatment of brain tumors sensitive to cytostatics, which are substrates of P-gp. The chemotherapy of other cancers expressing the MDR phenotype (e.g., colon carcinoma and kidney cancer) could also benefit from the coadministration of P-gp inhibitors such as valspodar. In the clinics, the risk of increased (CNS) toxicity, which cannot be totally excluded by our preclinical studies, might require an adjusted paclitaxel dose when used in combination with valspodar or other p-gp inhibitors such as elacridar.

\section{Acknowledgments}

We thank O. Gleich for his help in immunohistochemistry and F.Wiesenmayer, O. Baumann, P. Pistor, $\mathrm{K}$. Röhrl, and S. Bollwein for technical assistance. This study was supported by: Deutsche Forschungsgemeinschaft grants FR1211/6-1 and FR1211/6-2 (G. Fricker) and BU1084/1-2 (A. Buschauer); Forschungsschwerpunktsprogramm, Baden-Württemberg, K-170200/ 00.001 (G. Fricker); Fonds der Chemischen Industrie (A. Buschauer and G. Bernhardt).

1. Kroger, N., Achterrath, W., Hegewisch-Becker, S., Mross, K., and Zander, A.R. 1999. Current options in treatment of anthracycline-resistant breast cancer. Cancer Treat. Rev. 25:279-291.

2. Nathan, F.E., Berd, D., Sato, T., and Mastrangelo, M.J. 2000. Paclitaxel and tamoxifen: an active regimen for patients with metastatic melanoma. Cancer. 88:79-87.

3. Muggia, F.M, et al. 2000. Phase III randomized study of cisplatin versus paclitaxel versus cisplatin and paclitaxel in patients with suboptimal stage III or IV ovarian cancer: a gynecologic oncology group study. J. Clin. Oncol. 18:106-115.

4. Papadimitriou, C.A. 2000. Paclitaxel, cisplatin, and epirubicin first-line chemotherapy in stage III and IV ovarian carcinoma: long-term results of a phase II study. Cancer. 89:1547-1554.

5. Belani, C.P. 2000. Paclitaxel and docetaxel combinations in non-small cell lung cancer. Chest. 117(Suppl 1):144S-151S.

6. Bunn, P.A, Jr., and Kelly, K. 2000. New combinations in the treatment of lung cancer: a time for optimism. Chest. 117(Suppl 1):138S-143S.

7. Lee, J.S., et al. 1997. Paclitaxel/carboplatin chemotherapy as primary treatment of brain metastases in non-small cell lung cancer: a preliminary report. Sem. Oncol. 24(4 Suppl. 12):S12/52-S12/55.

8. Glantz, M.J., Chamberlain, M.C., Chang, S.M., Prados, M.D., and Cole, B.F. 1999. The role of paclitaxel in the treatment of primary and metastatic brain tumors. Sem. Rad. Oncol. 9:27-33.

9. Brandes, A.A., Pasetto, L.M., and Monfardini, S. 2000. New drugs in recurrent high grade gliomas. Anticancer Res. 20:1913-1920.

10. Eiseman, J.L., et al. 1994. Plasma pharmacokinetics and tissue distribution of paclitaxel in CD2F1 mice. Cancer Chemother. Pharmacol. 34:465-471.

11. Heimans, J.J., et al. 1994. Paclitaxel (taxol) concentrations in brain tumor tissue. Ann. Oncol. 5:951-953.

12. Sparreboom, A., et al. 1997. Limited oral bioavailability and active epithelial excretion of paclitaxel (taxol) caused by P-glycoprotein in the intestine. Proc. Natl. Acad. Sci. USA. 94:2031-2035.

13. van Asperen, J., et al. 1997. Enhanced oral bioavailability of paclitaxel in mice treated with the P-glycoprotein blocker SDZ PSC 833. Brit. J. Cancer. 76:1181-1183.

14. Martin, C., et al. 2000. Communication between multiple drug binding sites on P-glycoprotein. Mol. Pharmacol. 58:624-632.

15. Beaulieu, E., Demeule, M., Ghitescu, L., and Beliveau, R. 1997. P-glycoprotein is strongly expressed in the luminal membranes of the endothelium of blood vessels in the brain. Biochem. J. 326:539-544.

16. Miller, D.S., Nobmann, S., Gutmann, H., Drewe, J., and Fricker, G. 2000. Xenobiotic transport in isolated brain microvessels studied by confocal microscopy. Mol. Pharmacol. 58:1357-1367.

17. Seetharaman, S., Barrand, M.A., Maskell, L., and Scheper, R.J. 1998. Multidrug resistance-related transport proteins in isolated human brain 
microvessels and in cells cultured from these isolates. J. Neurochem. 70:1151-1159.

18. Nobmann, S., Bauer, B., and Fricker, G. 2001. Ivermectin excretion by isolated functionally intact brain endothelial capillaries. Brit. J. Pharmacol. 132:722-728.

19. Ohnishi, T., et al. 1995. In vivo and in vitro evidence for ATP-dependency of P-glycoprotein-mediated efflux of doxorubicin at the blood-brain barrier. Biochem. Pharmacol. 49:1541-1544.

20. Huwyler, J., Drewe, J., Kluseman, C., and Fricker, G. 1996. Evidence for P-glycoprotein-modulated penetration of morphine-6-glucuronide into brain capillary endothelium. Brit. J. Pharmacol. 118:1879-1885.

21. Drewe, J., et al. 1999. HIV protease inhibitor ritonavir: A more potent inhibitor of p-glycoprotein than the cyclo-sporine analogue SDZ PSC833. Biochem. Pharmacol. 57:1147-1152.

22. Schinkel, A.H., et al. 1994. Disruption of the mouse mdr1a P-glycoprotein gene leads to a deficiency in the blood-brain barrier and to increased sensitivity to drugs. Cell. 77:491-502.

23. Mayer, A., et al. 1997. Full blockade of intestinal P-glycoprotein and extensive inhibition of blood-brain barrier P-glycoprotein by oral treatment of mice with PSC833. J. Clin. Invest. 100:2430-2436.

24. Miller, D.S., and Pritchard, J.B. 1991. Indirect coupling of organic anion secretion to sodium in teleost (Paralichthys lethostigma) renal tubules. Am. J. Physiol. 261:R1470-R1477.

25. Schramm, U., Fricker, G., Wenger, R., and Miller, D.S. 1995. p-Glycoprotein mediated transport of a fluorescent cyclosporin analogue in teleost proximal tubules. Am. J. Physiol. 268:F46-F52.

26. Gutmann, H., et al. 1999. Modulation of MRP expression in porcine brain capillary endothelial cells in vitro. Drug Metab. Dispos. 27:937-941.

27. Audus, K. L., and Borchardt, R.T. 1986. Characterization of an in vitro blood-brain barrier model system for studying drug transport and metabolism. Pharm. Res. 3:81-87.

28. Bernhardt, G., Reile, H., Birnböck, H., Spruss, T., and Schönenberger, H 1992. Standardized kinetic microassay to quantify differential chemosensitivity based on proliferative activity. J. Cancer Res. Clin. Oncol. 118:35-43.

29. Reile, H., Birnböck, H., Bernhardt, G., Spruss, T., and Schönenberger, H. 1990. Computerized determination of growth kinetic curves and doubling times from cells in microculture. Anal. Biochem. 187:262-267.

30. Spruss, T., Schlemmer, R., Bernhardt, G., Wiesenmeyer, F., and Baumann, O. 1996. Five-year breeding data, immunology, and tumor take rates of an NMRI nude mouse colony. In Immunodeficient animals: model for cancer research. Volume 51. W. Arnold, P. Köpf-Maier, B. Michael, editors. $145-150$.

31. Sparreboom, A., van Tellingen, O., Nooijen, W.J., and Beijnen, J.H. 1995. Determination of paclitaxel and metabolites in mouse plasma, tissues, urine and faeces by semi-automated reversed-phase high-performance liquid chromatography. J. Chromatogr. B. 664:383-391.

32. Fellner, S., et al. 2000. The MDR modulator SDZ PSC 833 increases paclitaxel concentrations in the brain of nude mice. Arch. Pharm. Pharm. Med. Chem. 333(Suppl. 2):42. (Abstr.)

33. Romeis, B. 1989. Mikroskopische Technik. P. Böck, editor. Urban \& Schwarzenberg. Munich, Germany. 497-499.

34. Cordon-Cardo, C. 1991. Immunhistochemical analysis of p-glycoprotein expression in normal and tumor tissues in humans. In Molecular and cellular biology of multidrug resistance in tumor cells. Plenum Press. New York, New York, USA. 310-315

35. Fricker, G., Gutmann, H., Droulle, A., Drewe, J., and Miller, D.S. 1999.
Epithelial transport of anthelmintic ivermectin in a novel model of isolated proximal kidney tubules. Pharm. Res. 16:1570-1575.

36. Fracasso, P.M., et al. 1995. Phase I trial of paclitaxel (taxol) and SDZ PSC 833 in patients with solid tumors. Proc. Am. Soc. Clin. Oncol. 14:486a. (Abstr.)

37. Fracasso, P.M., et al. 2000. Phase I study of paclitaxel in combination with a multi drug resistance modulator, PSC 833 (Valspodar), in refractory malignancies. J. Clin. Oncol. 18:1124-1134.

38. Goldstein, L.J. 1996. MDR1 gene expression in solid tumors. Eur. J. Cancer. 32A:1039-1050.

39. Oda, Y., et al. 1997. Expression of MDR1/p-glycoprotein and multidrug resistance-associated protein in childhood solid tumours. Virchows Arch. 430:99-105.

40. Abe, T., et al. 1998. Expression of multidrug resistance protein gene in patients with glioma after chemotherapy. J. Neurooncol. 40:11-18.

41. von Bossanyi, P., Diete, S., Dietzmann, K., Warich-Kirches, M., and Kirches, E. 1997. Immunhistochemical expression of p-glycoprotein and gluthathione S-transferase in cerebral gliomas and response to chemotherapy. Acta Neuropathol. (Berl.). 94:605-611.

42. Scheper, R.J., et al. 1988. Monoclonal antibody jsb-1 detects a highly conserved epitope on the p-glycoprotein associated with multi-drug-resistance. Int. J. Cancer. 42:389-394.

43. Henson, J.W., Cordon-Cardo, C., and Posner, J.B. 1992. P-glycoprotein expression in brain tumors. J. Neurooncol. 14:37-43.

44. Toth, K., Vaughan, M.M., Peress, N.S., Slocum, H.K., and Rustum, Y.M. 1996. MDR1 P-glycoprotein is expressed by endothelial cells of newly formed capillaries in human gliomas but is not expressed in the neovasculature of other primary tumors. Am. J. Pathol. 149:853-858.

45. Sawada, T., Kato, Y., Sakayori, N., Takekawa, Y., and Kobayashi, M. 1999. Expression of multidrug-resistance P-glycoprotein (Pgp, MDR-1) by endothelial cells of the neovasculature in central nervous system tumors. Brain Tumor Pathol. 16:23-27.

46. Schaffrik, M. 2000. Untersuchungen zur Multi-Drug-Resistenz orthotoper menschlicher Hirntumoren in der Nacktmaus: Expression und Funktionalität des P-Glykoproteins 170 . Diploma thesis. University of Regensburg, Regensburg, Germany.

47. Altenschöpfer, P. 1998. Tumorpharmakologische und analytische Untersuchungen zur Optimierung neuer Zytostatika-beladener bioabbaubarer Implantate für die interstitielle Chemotherapie maligner Hirntumoren. Ph.D. thesis. University of Regensburg, Regensburg, Germany.

48. Altenschöpfer, P., Spruss, T., Bernhardt, G., and Buschauer, A. Comparison of subcutaneously and orthotopically implanted glioblastomas. Paper presented at: International Symposium: Relevance of Tumor Models for Anticancer Drug Development; October 15-17, 1997; Freiburg, Germany. (Abstr.)

49. Kreis, W., Budman, D.R., and Calabro, A. 2001. A reexamination of PSC 833 (Valspodar) as a cytotoxic agent and in combination with anticancer agents. Cancer Chemother. Pharmacol. 47:78-82.

50. Arnold, S.M., and Patchell, R.A. 2001. Diagnosis and management of brain metastases. Hematol. Oncol. Clin. North Am. 15:1085-1107.

51. Zhang, Y., Han, H., Elmquist, W.F., and Miller, D.W. 2000. Expression of various multidrug resistance-associated protein (MRP) homologues in brain microvessel endothelial cells. Brain Res. 876:148-153.

52. van Asperen, et al. 1996. Altered pharmacokinetics of vinblastine in MDR1a P-glycoprotein-deficient mice. J. Natl. Cancer Inst. 88:994-999.

53. Drion, N., Lemaire, M., Lefauconnier, J.M., and Scherrmann, J.M. 1996. Role of P-glyco-protein in the blood-brain transport of colchicine and vinblastine. J. Neurochem. 67:1688-1693. 